\title{
Inclusión Educativa en Síndrome de Asperger.
}

(Educational Inclusion in Asperger's syndrome)

\author{
Ana Lara Gómez \\ Universidad de Jaén, España.
}

Fecha recepción: 30-01-2018

Páginas 19-28

Fecha aceptación: 30-06-2018

\section{Resumen.}

Esta investigación trata sobre los procesos de inclusión del alumnado con Síndrome de Asperger.

Para su realización, se ha llevado a cabo un marco teórico que explica los conceptos básicos referentes al tema que ocupa, véase "Inclusión Educativa" y "Síndrome de Asperger". También se ha realizado un marco empírico en el que se detallan los puntos a seguir para la realización de la investigación: Problema a estudiar, objetivos (generales y específicos), hipótesis, contextualización, población y muestra, tipo de investigación, metodología, diseño de intervención, instrumentos para el estudio y resultados y análisis de los mismos, a fin de lograr obtener conclusiones sobre el problema planteado.

La investigación se llevó a cabo en un centro de la provincia de Jaén, a través de una escala Likert. Esta escala se repartió entre el profesorado de Educación Infantil a fin de observar y determinar el proceso de inclusión del alumnado con Síndrome de Asperger.

Palabras clave: Síndrome de Asperger; inclusión; necesidades específicas de apoyo educativo; discapacidad; investigación

\section{Abstract.}

This document presents a research about the different processes for the inclusion of students with Asperger's syndrome.

This document has been developed based on a theoretical framework which explains the basics concepts regarding the "Educational Inclusion" and "Asperger's Syndrome".

The aims of this document is the development of an empirical framework which describes the steps for carrying out the research. The steps are: problems statement, goals intended, description of the hypothesis, contextualization of the research, population and samples to be analyzed, kind of research, background, the methodology researching instruments and the analysis of the results.

The research has been developed in a centre in Jaen using as a base tool the Likert scalas. These scalas were shared with the teachers with the aim of inspect and determine the process of inclusion of students with Aspeger's syndrome.

Keywords: asperger's syndrome; inclusion; special needs for educational support, disability; research 


\section{1.-Introducción.}

Según la Real Constitución Española (1978), en su artículo 27 establece que: "Todos tienen derecho a la educación (...) La educación tendrá por objetivo el pleno desarrollo de la personalidad humana en el respeto de los principios democráticos de convivencia y a los derechos y libertades fundamentales." (p.14)

Ante una sociedad en constante cambio, cada vez existe más heterogeneidad en las aulas de los centros educativos, niños y niñas con características muy diferentes entre se reúnen cada día y pasan una gran cantidad de horas con sus profesores/as, los cuales tienen que hacerse cargo de un aula de 25 alumnos/as intentando dar una atención individualizada a cada estudiante. Es por ello que es necesario saber si todo el alumnado se siente integrado en el aula y que nunca sus diferencias sean motivo de rechazo por parte del sistema educativo.

Para evitar que ningún niño ni ninguna niña se sientan abandonados o fallados en su aula, es necesaria una educación con unos principios inclusivos, tal y como dice Amaro (2013): "La escuela inclusiva tiene como objetivo último que todo el alumnado desarrolle al máximo todas sus capacidades y potenciales para que puedan hacerse competentes en la construcción de su proyecto personal y profesional" (p. 53), de este modo la escuela tiene como deber hacer que cualquier alumno/a, independientemente de sus características personales, conseguir desarrollar al máximo su potencial. Así pues, el alumnado con necesidades educativas especiales debe pertenecer a un modelo de enseñanza-aprendizaje inclusivo y que se adapte a sus necesidades.

Esta investigación tiene como fin indagar sobre la percepción del profesorado de los niños y niñas con Síndrome de Asperger de Educación Infantil sobre los procesos de inclusión en el aula.

\section{2.-Marco teórico.}

Una delimitación conceptual es fundamental para entender las bases de una investigación, por lo que en los siguientes puntos recogidos en el marco teórico se pondrán encontrar las definiciones de los conceptos más importantes que atañen la investigación.

\section{1.-Inclusión Educativa.}

Acorde al pensamiento de Echeita y Ainscow (2011), la inclusión educativa, pese a su gran dificultad de una definición global, tiene como fin la eliminación de la exclusión social por la causa que fuere del sistema educativo, dando respuesta a la diversidad de nuestro alumnado.

Estos mismos autores destacan 4 elementos esenciales a fin de aclarar la terminología de la inclusión:

Inclusión como proceso: El tiempo es un factor determinante para que le logre un cambio de mentalidad y se pueda responder positivamente a la diversidad.

El éxito de todo el alumnado como objetivo: Las voces de todos nuestros alumnos y alumnas han de ser escuchadas, permitiendo a todas las personas no sólo estar en el aula ordinaria, sino participar y ser miembros activos en ella. 
Eliminación de barreras: Ya sean estructurales, metodológicas, referente a creencias negativas hacia determinados grupos de personas, prejuicios...

La formación del profesorado en metodologías inclusivas es esencial para que este movimiento educativo pueda llegar a tener éxito (Durán y Giné, 2017), esto es debido porque se ha de realizar un estudio sobre las características principales individuales dentro de la diversidad del alumnado, así como la demanda que ofrece la inclusión a intensificación pedagógica por parte del centro educativo y, además, sin olvidar que una mejora en la formación docente implica directamente en una mejora del sistema educativo en sí.

\section{2.-Síndrome de Asperger}

Hasta los años 40, existía un enorme desconocimiento hacia el autismo, el cual se consideraba retraso profundo, fue en 1943, cuando el psiquiatra estadounidense Leo Kanner realizó un extraordinario descubrimiento referente a un grupo de niños autistas: Eran inteligentes. Observó que algunos tenían gran vocabulario, otros eran superdotados para el cálculo, y todos poseían una gran memoria. Sin embargo, también afirmó su incapacidad biológica de desarrollar contactos afectivos. Un año después, en 1944, el neuropediatra vienés Hans Asperger realizó las primeras descripciones de lo que él llamó Psicopatía Autista, encontrándose muy cerca (cronológicamente hablando) con la publicación de Kanner sobre autismo.

En su informe, Asperger (1944) destacaba las siguientes características de la Psicopatía Autista en la niñez:

Dificultad en el contacto visual; Habla nasal, algunas veces demasiado suave y otras veces inapropiadamente alta, caracterizándose por un carácter monótono; Lenguaje excesivamente elaborado, uso idiosincrásico de la lengua, dificultad para comprender las frases con significados literales; Intereses obsesivos; Tendencia al aislamiento y dificultad para mantener relaciones sociales; Dificultad para mantener la atención y la concentración; Carácter irritable; Pensamientos raros; Torpeza motora.

El trabajo de Hans Asperger no tuvo mayor relevancia hasta que, en 1991, Uta Frith tradujese al inglés su trabajo escrito en alemán, cobrando en ese momento el estudio de Asperger mayor notoriedad. El término "Síndrome de Asperger" fue dado por Lorna Wing en 1981 ya que se trataba de un término neutro y a fin de evitar las connotaciones negativas que podrían asociarse al término "psicopatía autista".

Lorna Wing (1981) recogió los principales rasgos clínicos descritos originalmente por Asperger, siendo estos:

Lenguaje: Se observaron diferentes características en esta área, véase: Dificultad en el uso de pronombres, discurso pedante y enfocado a los intereses de la persona, repetición de frases o palabras e incapacidad o dificultad para entender bromas; Lenguaje no verbal: Escasez de comunicación corporal y gesticulación facial, entonación monótona, limitada comprensión de los gestos ajenos y expresiones, así como su interpretación; Interacción social: Falta de contacto visual en las interacciones sociales, ausencia de la habilidad para acatar normas, incomprensión para saber mantener relaciones sociales, sensibilidad a las críticas, comportamiento antisocial o peculiar; Frustración ante el cambio y disfrute con las actividades repetitivas; Torpeza motora, movimientos corporales estereotipados, postura 
inadecuada, mala escritura; Excelente memoria para sus temas de interés, siendo intensos y extraños en relación a su edad.

Según la Asociación Americana de Psiquiatría, en el manual de diagnóstico del DSMV (2013), el Síndrome de Asperger está incluido dentro del Trastorno del Espectro Autista, modificando así la definición anteriormente dada por el DSM-IV (2000), que proclamaba que el síndrome de Asperger era parte de los cinco trastornos definidos como "Trastornos Generalizados del Desarrollo".

El Síndrome de Asperger es definido también por diferentes autores como BaronCohen (2008) que afirma que las características del Síndrome de Asperger se focalizan en la dificultad residente en la comunicación social, la existencia de intereses obsesivos y conductas rituales, sin apreciación de retrasos en la adquisición del lenguaje. Añade que el coeficiente intelectual no se ve afectado, se encuentra mínimo en la media, pudiendo ser mayor que ésta incluso.

Según Alonso (2004), en personas con este trastorno, también se aprecia, además, un pensamiento concreto y literal, es decir, existe una incapacidad para entender recursos lingüísticos como el sarcasmo, la ironía o las frases hechas. La obsesión con ciertos temas hace que sea evidente su excelente memoria, recordando cualquier dato sobre el mismo y volviéndose auténticos expertos en ese tema. Estas personas pueden funcionar a alto nivel, trabajar y vivir independientemente.

Para finalizar este apartado de conceptualización sobre el Síndrome de Asperger, mencionar que investigaciones como la llevada a cabo por Cejudo y Corchuelo (2018), afirman que una intervención y evaluación psicopedagógica será esencial para el desarrollo personal y futuro laboral del alumnado con síndrome de Asperger.

\section{3.-Marco empírico.}

Nuestra investigación es de naturaleza exploratoria y descriptiva, adoptaremos una metodología cuantitativa, pues nuestro propósito es concluir si existe un proceso de inclusión real del alumnado de Educación Infantil con Síndrome de Asperger.

\section{2.-Metodología.}

La metodología que se va a llevar a cabo en esta investigación consiste en un enfoque cuantitativo.

Es un proceso riguroso, cuidadoso y sistematizado en el que se busca resolver problemas, bien sea de vacío de conocimiento o de gerencia, pero en ambos casos es organizado y garantiza la producción de conocimiento 0 de alternativas de solución viables.

El modelo cuantitativo hace que el investigador, por un lado, crea poder explicar, en una visión racionalista, su objeto de estudio; solo exteriormente, sin dialogar con él, sin interactuar con él, por otro lado, del punto de vista relativista, fundamentado en la epistemología subjetivista, por entender que hay múltiples realidades que existen bajo forma de construcciones mentales y socialmente localizadas por la comprensión, significado y acción. Pensamientos estos que iluminaron por varias décadas, la definición de los pasos que los investigadores recorrían en sus estudios y que acabaron reflejándose siempre en las prácticas escolares, en las pedagogías dominante y en la educación. 
El enfoque cuantitativo es adecuado para esta investigación, ya que la cuantificación es un hecho que tendremos en cuenta para comprender la realidad estudiada.

\section{3.-Problema a estudiar.}

¿Los procesos de inclusión en los centros educativos actuales son realmente efectivos con el alumnado de Síndrome de Asperger.?

\section{4.-Objetivos.}

Generales: Analizar la percepción que tienen el profesorado del alumnado con Síndrome de Asperger de la etapa de Educación Infantil sobre el proceso de inclusión en el aula.

Específicos:

1)Observar si el profesorado del alumno con Síndrome de Asperger comprende qué es la educación inclusiva.

2)Estudiar si el profesorado del alumnado con Síndrome de Asperger entiende el significado de "personas con diversidad funcional" (discapacidad).

3)Observar si el profesorado del alumnado con Síndrome de Asperger conoce los Trastornos del Espectro Autista.

4)Verificar que el profesorado del alumnado con Síndrome de Asperger es consciente de lo que es el Síndrome de Asperger.

5)Confirmar si existen procesos de inclusión en el aula del alumnado con Síndrome de Asperger.

\section{5.-Hipótesis.}

Hipótesis 0: Los procesos de inclusión en los centros educativos actuales si son realmente efectivos con el alumnado de Síndrome de Asperger.

Hipótesis 1: Los procesos de inclusión en los centros educativos actuales no son realmente efectivos con el alumnado de Síndrome de Asperger.

\section{6.-Población y muestra.}

La población susceptible de la investigación la forman el profesorado del alumnado con Síndrome de Asperger dentro de la etapa de Educación Infantil, siendo seleccionados de forma no probabilística intencional, dado que se pretende estudiar al profesorado que tiene alumnos con Síndrome de Asperger, siendo este el criterio de selección.

La población susceptible de ser investigada la forman docentes de Educación Infantil de un colegio de la provincia de Jaén, siendo un total de 14 docentes activos y que actualmente imparten clase. La población coincide con la muestra.

\section{7.-Instrumentos de recolección de datos.}

Para la realización de nuestro estudio, optamos por la utilización de una escala como instrumento de recogida de datos. Con toda probabilidad, la escala de categorías constituye una de las técnicas de medida de creencias, preferencias y actitudes más utilizada por los científicos de la conducta. 
La encuesta es de tipo Escala Likert, consistiendo en 25 preguntas en relación a los objetivos específicos de la investigación. Murillo (2006) define la mencionada escala como un medio capaz de reflejar las actitudes que siente el encuestado hacia un tema, ya sea de una forma positiva o negativa, calificando en función de cuánto de acuerdo esté con la afirmación.

En función de los objetivos específicos y del análisis de marco teórico las dimensiones que estructuran la escala Likert son:

Dimensión 1.- Inclusión Educativa

Dimensión 2.- Personas con Diversidad Funcional

Dimensión 3.- Trastornos del Espectro Autista

Dimensión 4.- Síndrome de Asperger

Dimensión 5.- Proceso de inclusión del alumno/a con Síndrome de Asperger

3.8.-Análisis e interpretación de los datos.

A continuación, se mostrará un análisis de los resultados obtenidos en la investigación realizada, así como una interpretación sobre los mismos, en relación con los objetivos específicos planteados.

\subsection{1.-Dimensión 1. Inclusión Educativa.}

Esta dimensión se relaciona con el objetivo específico: 1) Observar si el profesorado del alumno con Síndrome de Asperger comprende qué es la educación inclusiva.

Los y las docentes encuestados están familiarizados con el movimiento de la educación inclusiva. Si bien existe todavía un pequeño porcentaje de aquel profesorado que mantiene una mentalidad de segregación e integración, una amplia mayoría apuesta por la inclusión como meta educativa a seguir, conociendo sus pilares fundamentales y llevándolos a cabo en sus aulas.

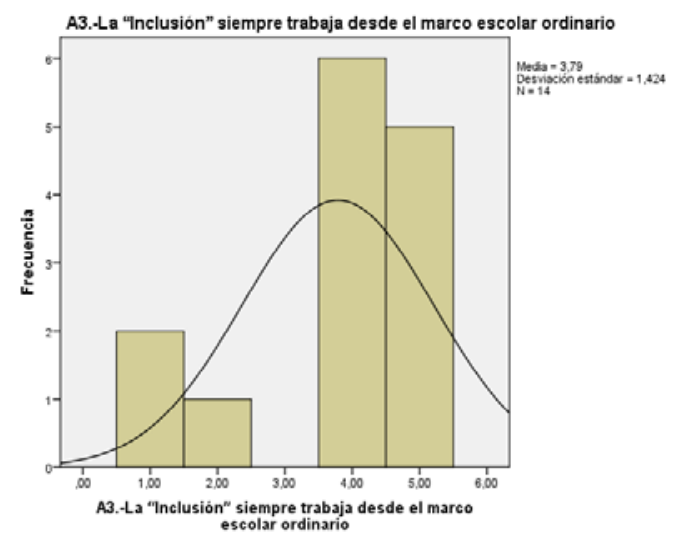

Gráfica 1: Ejemplo gráfica dimensión 1.

3.8.2.-Dimesión 2. Diversidad Funcional.

Esta dimensión se relaciona con el objetivo específico: 2) Estudiar si el profesorado del alumnado con Síndrome de Asperger entiende el significado de "personas con diversidad funcional" (discapacidad). 
En relación con este objetivo y a través de los datos recolectados, se podría decir que, aunque no mayoritario, un porcentaje del profesorado todavía no comprende en qué consiste la diversidad funcional, y por lo tanto muestra una respuesta desviadas de su inclusión en el aula ordinal.



Gráfica 2: Ejemplo gráfica dimensión 2.

\subsection{3.-Dimensión 3. Trastornos del Espectro Autista.}

Esta dimensión se relaciona con el objetivo específico: 3) Observar si el profesorado del alumnado con Síndrome de Asperger conoce los Trastornos del Espectro Autista. Los datos obtenidos son muy positivos frente a este objetivo. La gran mayoría del profesorado conoce los Trastornos del Espectro Autista y sus características principales, siendo esto un factor determinante para su inclusión en el aula.

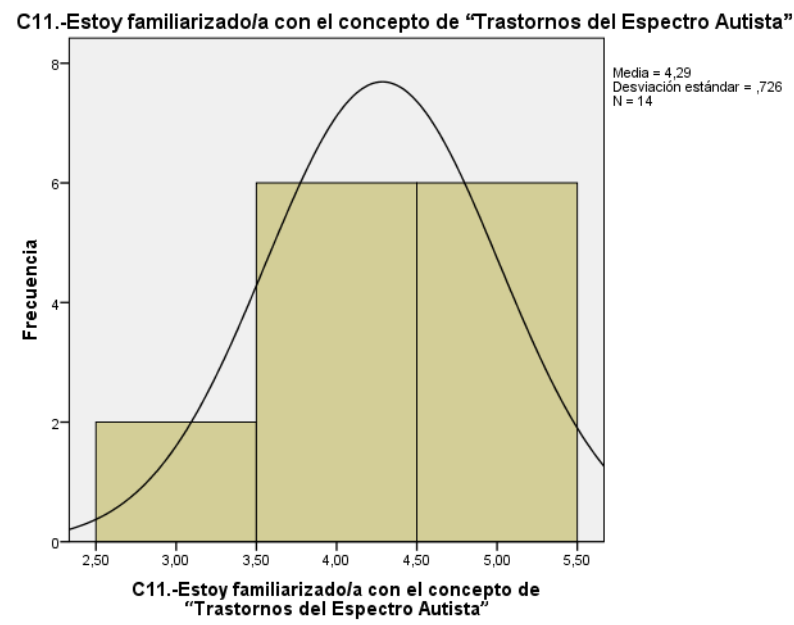

Gráfica 3: Ejemplo gráfica dimensión 3. 
3.8.4.-Dimensión 4. Síndrome de Asperger.

Esta dimensión se relaciona con el objetivo específico: 4) Verificar que el profesorado del alumnado con Síndrome de Asperger es consciente de lo que es el Síndrome de Asperger.

Los datos obtenidos confirman que el profesorado entiende y conoce las características principales del Síndrome de Asperger, siendo un factor indispensable para lograr su inclusión.

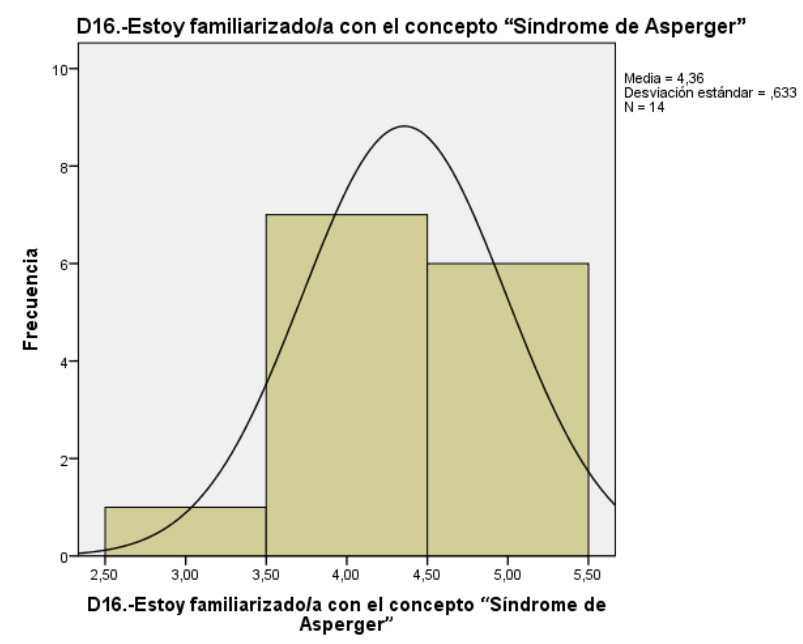

Gráfica 4: Ejemplo gráfica dimensión 4.

3.8.5.-Dimensión 5. Proceso de inclusión del alumno/a con Síndrome de Asperger Esta dimensión se relaciona con el objetivo específico: 5) Confirmar si existen procesos de inclusión en el aula del alumnado con Síndrome de Asperger.

Mediante el estudio de todos los datos obtenidos, se puede llegar a la conclusión de que existen procesos de inclusión reales con el alumnado con Síndrome de Asperger. Una mayoría del profesorado apuesta por sistemas educativos inclusivos para sus alumnos con Síndrome de Asperger, llevando a cabo metodologías, evaluaciones y respuestas educativas específicas para cada alumno y alumna, formándose como docentes a fin de conocer y entender la diversidad de su alumnado, más concretamente, en referencia al alumnado con Síndrome de Asperger. 


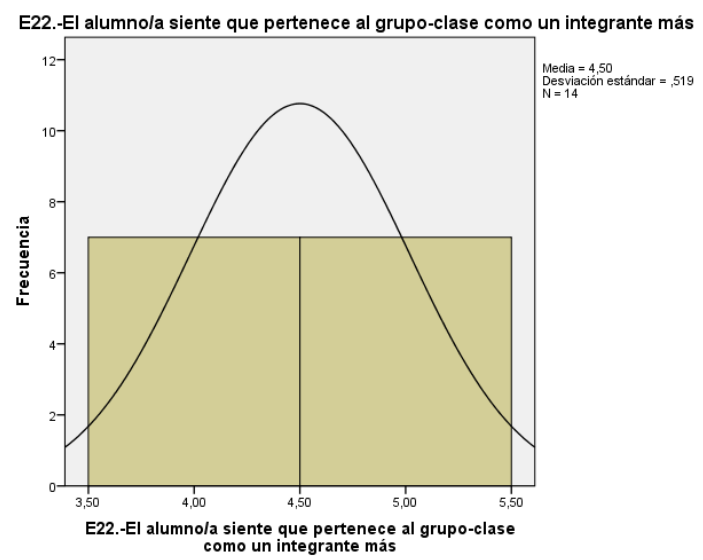

Gráfica 5: Ejemplo gráfica dimensión 5.

\section{4.-Conclusión.}

El marco teórico nos marcaba en el punto 5.1lnclusión Educativa, que la inclusión trata de que el sistema educativo se adapte a las necesidades y características del alumno/a, y no al contrario, siendo fundamental la inexistencia de actitudes de rechazo o discriminación hacia el alumnado con necesidades específicas de apoyo educativo, siendo el alumnado con Síndrome de Asperger en esta investigación. En base a esta definición dada de inclusión, podemos observar que, en la Dimensión 5 : Inclusión educativa del alumnado con Síndrome de Asperger, en los ítems del 20 hasta el 25, el profesorado afirma que su alumnado con Síndrome de Asperger no ha sufrido ninguna clase de rechazo en el centro escolar, y que éste además, modifica la metodología y los procesos de enseñanza-aprendizaje para dar una óptima respuesta educativa a este alumnado, cumpliéndose de este modo lo dictaminado en la definición de inclusión educativa.

En el punto 5.1 Inclusión Educativa, también se hacía referencia a la necesidad de una correcta formación docente, algo imprescindible para que se puedan lograr cumplir los procesos de inclusión. En relación a este requerimiento, y siguiendo la información recogida en el punto 5.3 Síndrome de Asperger, se observa en la Dimensión 4: Síndrome de Asperger que el profesorado encuestado conoce el Síndrome de Asperger y sus características principales.

En relación con lo explicado se puede decir entonces que lo dictaminado en el marco teórico es cumplido según lo compilado en el marco empírico.

A través del análisis e interpretación de los datos, podemos concluir lo siguiente:

La percepción sobre el proceso de inclusión en el aula que tienen el profesorado del alumnado con Síndrome de Asperger en la etapa de Educación Infantil es positiva, cumpliéndose así pues la hipótesis 0: Los procesos de inclusión en los centros educativos actuales si son realmente efectivos con el alumnado de Síndrome de Asperger. 


\section{5.-Bibliografía.}

Alonso, J.R. (2004). Autismo y Síndrome de Asperger. Guía para familiares, amigos y profesionales. Salamanca: Editoriales Amarú.

Amaro, A.E. (coord.). (2013). Atención a la diversidad en el aula de Educación Infantil. Inclusión del alumnado con NEAE. Madrid: Editorial Universitas, S.A.

American Psychiatric Association. (2002). Manual Diagnóstico y Estadístico de los Trastornos Mentales DSM-IV-TR. Barcelona: Editorial Masson.

American Psychiatric Association. (2014). Manual Diagnóstico y Estadístico de los Trastornos Mentales DSM-V-TR. Madrid: Editorial Médica Panamericana.

Baron-Cohen, S. (2008). Autismo y Síndrome de Asperger. Madrid: Editorial Alianza.

Cejudo, A. y Corchuelo, C. (coords.) (2018). La evaluación psicopedagógica a debate. Reflexiones y experiencias de profesionales de titulaciones educativas. Sevilla: AFOE.

Durán, D. y Giné, G. (2017). La formación del profesorado para la educación inclusiva: Un proceso de desarrollo profesional y de mejora de los centros para atender la diversidad. Red iberoamericana de expertos en la convención de los derechos de las personas con discapacidad.153-170.Recuperado de http://repositoriocdpd.net:8080/handle/123456789/1913

Echeita, G. y Ainscow, M. (2011). La educación inclusiva como derecho. Marco de referencia y pautas de acción para el desarrollo de una revolución Murillo, $F$. J. (2006). Cuestionarios y escalas de actitudes. Madrid: UAM. pendiente. Tejuelo, $n^{0}$ 12, 26-46. 